\title{
Correlation between N-Terminal-Pro-Brain Natriuretic Peptide and Heart Failure in Children with Acyanotic Congenital Heart Disease Left-to-right Shunt
}

\author{
Rizka Arifani ${ }^{1}$, Mahrus A. Rahman ${ }^{1}$ Jusak Nugraha ${ }^{2}$ \\ official email address: arifanirizka @gmail.com \\ 1Child Health Department, Medical Faculty, Airlangga University/Dr. Soetomo General Hospital, Surabaya 60286-Indonesia \\ 2Clinical Pathology Department, Medical Faculty, Airlangga University/Dr. Soetomo General Hospital, Surabaya 60286-Indonesia
}

\begin{abstract}
Background: Heart failure is a significant problem causing morbidity and mortality in children with acyanotic CHD leftto-right shunt. Congenital heart disease is often associated with ventricular dysfunction, volume or pressure overload, resulting in a high risk of heart failure. N-Terminal-pro-Brain Natriuretic Peptide (NT- pro-BNP) is a biochemical marker in the left ventricle, which is used as a diagnostic marker for adult heart failure since it has depicted a significant correlation between heart failure and elevated levels of NT-pro-BNP. This study aimed to analyze the relationship between NT-pro-BNP levels and heart failure in children with acyanotic CHD left-to-right shunt.
\end{abstract}

Methods: An observational analytic examination with a cross-sectional study design was conducted on children aged 1 month to 18 years with acyanotic CHD left-to-right shunt with suspected heart failure at the Pediatric Cardiology Outpatient Clinic, Emergency Department, and Pediatric Ward of Dr. Soetomo General Hospital Surabaya from September 2020 to March 2021. Statistical analysis was carried out using Mann-Whitney test and contingency correlation test of Chi-Square with a significance value of $p<0.05$.

Results: There were 43 subjects fulfilling the inclusion criteria in which 53.5\% of them were female with a mean age of 48 months. Furthermore, the most common type of acyanotic CHD left-to-right shunt with heart failure was VSD, followed by ASD and PDA. There was a significant relationship between increasing NT-pro-BNP levels with heart failure with $p<0.001$ based on chi-square test with moderate correlation strength $(r=0.57)$ based on the contingency correlation test.

Conclusion: There is a significant positive correlation between NT-pro-BNP and heart failure in children with acyanotic CHD left-to-right shunt.

Keywords: NT-pro-BNP; acyanotic CHD; Heart failure 


\section{Introduction}

The case of congenital heart disease (CHD) among newborns ranges from about 3.7 to 17.5 per 1000 cases, $30-45 \%$ of all congenital abnormalities [1]. Patients with the acyanotic CHD left-to-right shunt are at risk more than 100 times for heart failure [2]. There are 12,000-35,000 cases of heart failure causing 11,00014,000 hospitalizations in children younger than 19 years in the United States each year [3].

Congenital heart disease is often associated with ventricular dysfunction, volume or pressure overload, resulting in a high risk of heart failure [4] As a compensatory mechanism for pressure and volume overload, Brain Natriuretic Peptide (BNP) and N-Terminal-pro-Brain Natriuretic Peptide (NT-pro-BNP) are secreted in the left ventricle [5]. In adult, NT-pro-BNP levels have been used as a diagnostic marker for heart failure because it has portrayed a significant correlation between heart failure and increasing levels of NT-pro-BNP [6]. To the best of the researchers' knowledge, studies on the role of NT-pro-BNP in the diagnosis of heart failure in children have not been discussed yet. This study aimed to analyze the relationship between NT-proBNP levels and heart failure in children with acyanotic CHD CHD left-to-right shunt.

\section{Subjects and Methods}

\subsection{Study Design}

The present study used an analytical observational research approach with a cross-sectional design. This study analyzed the correlation between NT-pro-BNP and heart failure in children with acyanotic CHD. The subjects were all children aged 1 month - 18 years old with acyanotic CHD left-to-right shunt treated at the Division of Pediatric Cardiology, Dr Soetomo general hospital Surabaya. The subjects were selected by consecutive non-random sampling technique. The study was approved by the Ethical Committee of Dr Soetomo Academic General Hospital (No. 2032 / KEPK / IX / 2020).

\subsection{Diagnosis of Acyanotic CHD and Heart Failure}

The definite diagnosis of congenital heart disease was determined by the basis of an echocardiography examination performed by a pediatric cardiologist. The types of heart defects classified as acyanotic CHD were atrial septal defect (ASD), ventricle septal defect (VSD), and patent ductus arteriosus (PDA). The diagnosis of heart failure was based on Pediatric Heart Failure Score, which became the standard for diagnosing heart failure in children.

\subsection{Laboratory Examination}

About 2-3ml of venous blood from children with acyanotic CHD left to right shunt suspected of heart failure was obtained and tested. The blood placed in a yellow vacutainer tube containing the separator gel was centrifuged to obtain serum. The serum was then stored at $-80 \mathrm{oC}$ and measured by the IMMULITE 1000 Turbo NT-pro-BNP test kit.

\subsection{Statistical analysis}

Statistical measures (e.g. mean, standard deviation, and frequency distribution table) were presented in the form of descriptive analysis. The data were processed using SPSS version 21.0. The correlation between NT-pro-BNP and heart failure was analyzed by Mann-Whitney test and contingency correlation test of Chi-Square. 


\section{Results}

3.1 Characteristic of patients

The subjects were 43 children with acyanotic CHD left to right shunt aged from 1 month to 18 years. Table 1 shows characteristic of patients. [Table 1]

Table 1. Characteristic of Subject

\begin{tabular}{|c|c|}
\hline Characteristic & $\mathrm{n}=43$ \\
\hline Age (month), median $(\min -\max )$ & $48(2-201)$ \\
\hline \multicolumn{2}{|l|}{ Age group (month), n (\%) } \\
\hline $0-12$ & $11(25,6)$ \\
\hline $13-83$ & $25(58,1)$ \\
\hline $84-131$ & $4(9,3)$ \\
\hline$>131$ & $3(7,0)$ \\
\hline \multicolumn{2}{|l|}{ Child sex, n (\%) } \\
\hline Male & $20(46,5)$ \\
\hline Female & $23(53,5)$ \\
\hline \multicolumn{2}{|l|}{ Acyanotic CHD type } \\
\hline VSD & $17(39,5)$ \\
\hline ASD & $16(37,2)$ \\
\hline PDA & $10(23,3)$ \\
\hline \multicolumn{2}{|l|}{ Heart failure } \\
\hline Without heart failure & $9(21,0)$ \\
\hline Heart failure & $34(79,0)$ \\
\hline Mild & $8(19,0)$ \\
\hline Moderate & $12(28,0)$ \\
\hline Severe & $14(32,0)$ \\
\hline
\end{tabular}

3.2 Correlation between NT-pro-BNP and Heart Failure

This study showed a significant difference in NT-pro-BNP levels in children with acyanotic CHD left to right shunt who had heart failure and did not have heart failure $(\mathrm{p}<0.001)$. It was also found that there was a positive correlation between NT-pro-BNP levels and heart failure in children with acyanotic CHD left to right shunt $(\mathrm{r}=0.57)$. [Table 2]

Table 2. Correlation of NT-pro-BNP and heart failure in children with acyanotic CHD left to right shunt

\begin{tabular}{lllll}
\hline Criteria & mean & total & $\mathrm{p}$ & $\mathrm{r}$ \\
\hline Heart failure & $6549 \mathrm{pg} / \mathrm{ml}$ & 34 & $<0,001^{*}$ & $0,57^{* *}$ \\
Without heart failure & $234,36 \mathrm{pg} / \mathrm{ml}$ & 9 & & \\
\hline
\end{tabular}

*Mann-Whitney test

**Contingengsi correlation test 


\section{Discussion}

In accordance with this study's findings, heart failure in children with acyanotic CHD left to right shunt was more common in females than males, with a percentage of $53.5 \%$ and $46.5 \%$, respectively. A retrospective study conducted in China in 2016 also found that the prevalence of females was higher than that of males (52.7\% compared to $47.3 \%$ ) [7]. The case is possibly higher than in males because there are biological differences in the size of the structure of blood vessels, where the diameter of the arteries in female is smaller than in men. This condition increases the risk of vascular endothelial dysfunction, thereby contributing to the problem of pulmonary hypertension [8].

The present study also found that the age range for heart failure in children with CHD left to right shunt was 2 to 201 months, with a mean age of 48 months. The highest frequency of heart failure in this study was in the age group of 13 to 83 months. Differences in the age group for heart failure in children with acyanotic CHD left to right shunt are determined by the type of CHD and its complexity. The size of the defect will determine the magnitude of the shunt that occurs, which further affects the workload of the heart [9].

In addition, this study conveyed that the most common type of acyanotic CHD left to right shunt with heart failure was VSD (39.5\%), followed by ASD (37.2\%), and PDA (23.2\%). Heart failure in children with acyanotic CHD has the greatest prevalence due to VSD. It is related to the prevalence of VSD, which has been the most common type of CHD in children, and the size of the VSD lesion that affects the magnitude of the shunt and pulmonary vascular resistance [10].

In this study, there was a positive correlation between levels of NT-pro-BNP and the severity of heart failure in children with acyanotic CHD left to right shunt. The increase in NT-pro-BNP has a moderate correlation $(r=0.57)$. A cross-sectional study conducted in Medan, Indonesia, in 2015 in 30 children aged 1 month to 14 years found a significant correlation between levels of NT-pro-BNP and heart failure. There was a positive correlation between NT-pro-BNP levels and the severity of heart failure in children. In this study, it was not specific to heart failure caused by congenital heart disease. The test tool used Roche cardiac proBNP testing kit [11].

A study with a case-control design in 31 children with congestive heart failure also found that NT-pro-BNP levels had positively correlated with heart failure severity $(r=0.74)$. The severity of heart failure was assessed based on Pediatric Heart Failure Score, and the levels of NT-pro-BNP were measured using Enzyme Immuno Assay Biomedica-Austria. However, this study did not distinguish between heart failure caused by CHD or not CHD [12].

Furthermore, a study with a prospective design conducted in Japan, with an age range of 4 months to 14 years in 181 children with heart failure and 232 healthy children found a correlation between NT-pro-BNP levels and the severity of heart failure $(\mathrm{p}<0.001)$. The highest levels of NT-pro-BNP were found in children with severe heart failure. NT-pro-BNP levels were measured using Elecsys 2010 analyzer luminescent immunoassay kit (Roche Diagnostics; Mannheim, Germany). This study grouped children with heart failure into two age groups namely those $<3$ years and those $>3$ years. Unfortunately, this study was not limited to heart failure in CHD [13].

A study with a case-control design undertaken in Egypt on 40 children with heart failure found that increasing levels of NT-pro-BNP had a significant correlation with the severity of heart failure $(p<0.05)$. The more severe the severity of heart failure, the higher the mean value of NT-pro-BNP. However, this study was limited to children with acyanotic CHD in the form of a VSD with a mean age of 12 months. The examination of NT-pro-BNP was conducted by using Roche proBNP radioimmunoassay testing kit [14].

A cross-sectional study conducted in China on 80 children with heart failure found a significant relationship between increasing levels of NT-pro-BNP and the severity of heart failure $(p<0.001 ; r=0.675)$. The examination of NT-pro-BNP was conducted by using Canadian RAMP heart failure diagnostic 
instrument, Response Biomedical Corp. In this study, the median value of mild heart failure was $491 \mathrm{ng} / \mathrm{L}$, moderate heart failure of $3351 \mathrm{ng} / \mathrm{L}$, and severe heart failure of $20359 \mathrm{ng} / \mathrm{L}$. However, this study was not limited to heart failure caused by congenital heart disease [15].

Cardiac ventricular myocytes will hide NT-pro-BNP in response to an increase in ventricular wall pressure or an increase in ventricular volume, which cause stretching of the ventricular wall [16]. Brain natriuretic peptide and NT-pro-BNP levels reflect the hemodynamic status and pulmonary capillary pressure ventricular end-diastolic pressure. The more severe the degree of heart failure, the higher the pressure on the ventricular wall, of which this results in higher secretion of NT-pro-BNP by cardiac myocytes [17].

\section{Conclusion}

There is a significant positive correlation between NT-pro-BNP and heart failure in children with acyanotic CHD left to right shunt.

\section{Actknowledgement}

None.

\section{Financial Support}

This research received no specific grant from any funding agency, commercial or not-for-profit sectors.

\section{Conflict of Interest}

None.

\section{Ethical Standard}

This study obtained permission from the ethics committee. Ethical approval was obtained from the Institutional Ethical Committee of Dr. Soetomo general hospital (No. 2032 / KEPK / IX / 2020). All procedures performed were in accordance with the ethical standards and with the 1964 Helsinki Declaration and its later amendments. Before the subject recruitment, an explanation of general research information was carried out to the subjects and their parents for getting their consent. 


\section{References}

1. Sayasathid J, Sukonpan K, Sombo N. Epidemiology and etiology of congenital heart diseases. Congenit. Heart Dis 2012; 6: 139.

2. Gilljam T, Mandalenakis Z, Dellborg M et al. Development of heart failure in young patients with congenital heart disease: A nation-wide cohort study. Open Hear 2019; 6: 1-7.

3. Nandi D and Rossano JW. Epidemiology and cost of heart failure in children. Cardiol. Young 2015; 2030: 1460-68.

4. Rossano JW, Kim JJ, Decker JA, Price JF, Zafar F, Graves DE. Prevalence, morbidity, and mortality of heart failure-related hospitalizations in children in the United States: A population-based study. J. Card. Fail 2012; 18: 459-70.

5. Favilli S. Frenos S, Lasagni D et al. The use of B-type natriuretic peptide in paediatric patients : a review of literature. Fed. Ital. di Cardiol 2009;10: 298-302.

6. Eindhoven JA, Boersma E, and Roos-hesselink JW. The usefulness of brain natriuretic peptide in simple congenital heart disease - a systematic review. Cardiol. Young 2013; 23: 315-24.

7. Deng M, Lin C, Tang W, Zhu H, Zhang Y. Plasma N-terminal pro-B-type natriuretic peptide: Selecting the optimal heart failure marker in children of age up to 18 years. Int. J. Clin. Exp. Pathol 2016; 9:10756-62.

8. Verheugt CL, Uiterwaal CSPM, Van Der Velde ET et al. Gender and outcome in adult congenital heart disease. Circ. J 2008; 118: 26-32.

9. McDaniel NL. Ventricular and atrial septal defects. Pediatr. Rev 2001; 22: 265-70.

10. Hinton RB and Ware SM. Heart failure in pediatric patients with congenital heart disease. Circ. Res 2017; 120: 978-94.

11. Mahrani, Y., R. Nova, M.I. Saleh, and K.Y. Rahadianto. 2017. Correlation of heart failure severity and N-terminal pro-brain natriuretic peptide level in children. Paediatr. Indones. 56: 315-19.

12. Thomas M, Laer S, Eiselt M, and Grollmus O. Plasma Concentrations of N-Terminal pro-brain natriuretic peptide in control children from the neonatal to adolescent period and in children with congestive heart failure. Paediatrics 2002; 110: 1-8.

13. Sugimoto M, Manabe H, Nakau K et al. The role of N-Terminal Pro-B-Type Natriuretic Peptide in the diagnosis of congestive heart failure in children. Circ. J 2010; 74: 1-8.

14. Elsharawy S, Hassan B, Morsy S, Khalifa N. Diagnostic value of N-terminal pro-brain natriuretic peptide levels in pediatric patients with ventricular septal defect. Egypt. Hear. J 2012; 64: 241-46.

15. Lin CW, Zeng XL, Jiang SH et al. Role of the NT-proBNP level in the diagnosis of pediatric heart failure and investigation of novel combined diagnostic criteria. Exp. Ther. Med 2013; 6: 995-9.

16. Takei Y, Ando H, Tsutsui K. B-type Natriuretic Peptide. In: Ando, H., Tsutsui, K. (Ed.). Handbook of Hormones. Academic Press. Tokyo. 2015

17. Ka JKC, Janota T, Kasalová Z, and Hradec J. Natriuretic peptides - physiology, pathophysiology and clinical use in heart failure. Physiol Res 2009; 58: 171-7. 